\title{
Determination of the values of hole-mixing coefficients due to interface and electric field in $\mathrm{GaAs} / \mathrm{Al}_{x} \mathrm{Ga}_{1-x} \mathrm{As}$ superlattices
}

\author{
Xiao-Ling Ye, ${ }^{*}$ Y. H. Chen, J. Z. Wang, and Z. G. Wang \\ Laboratory of Semiconductor Materials Science, Institute of Semiconductors, Chinese Academy of Sciences, P.O. Box 912, \\ Beijing 100083, People's Republic of China \\ Z. Yang \\ Department of Physics, Hong Kong University of Science and Technology, Clearwater Bay, Kowloon, Hong Kong, China \\ (Received 8 May 2000; revised manuscript received 13 September 2000; published 1 March 2001)
}

\begin{abstract}
Numerical calculations within the envelope function framework have been performed to analyze the relations between the magnitude of in-plane optical anisotropy and the values of the additional hole-mixing coefficients due to interface and electric field in (001) symmetric GaAs/ $\mathrm{Al}_{x} \mathrm{Ga}_{1-x}$ As superlattices for light propagating along the [001] direction. It is found that the heavy- and light-hole states are mixed independently by interface and electric field. The numeric results demonstrate that the line shape of the in-plane anisotropic spectrum is determined by the ratio of the two hole-mixing coefficients. Theoretical analysis shows that with the help of simple calculation of the anisotropy at $k=0$, reliable values of the hole-mixing coefficients can be determined by reflectance-difference spectroscopy (RDS) technique, demanding no tedious fitting of experimental curves. The in-plane optical anisotropy measured by RDS provides a new method of getting the information on buried interfaces through the value of the hole-mixing coefficient due to interface.
\end{abstract}

DOI: 10.1103/PhysRevB.63.115317

PACS number(s): 78.66.Fd; 78.20.Bh; 78.20.Fm

\section{INTRODUCTION}

It is widely believed that [001]-grown zinc-blende-based superlattices have an isotropic linear optical response in the (001) plane. But giant in-plane optical anisotropy have been observed in $\mathrm{In}_{x} \mathrm{Ga}_{1-x} \mathrm{As} / \mathrm{InP}, \mathrm{In}_{x} \mathrm{Al}_{1-x} \mathrm{As} / \mathrm{InP}$, biased GaAs/ AlAs quantum wells (QW's), etc., for light propagating along [001] axis and their existences have been proved by symmetry arguments. ${ }^{1-9}$ It is found that the lower symmetry $\left(C_{2 v}\right)$ of the chemical bond arrangement for interface atoms can introduce asymmetry in [001] direction, which will induce anisotropy in (001) plane. Krebs et al. ${ }^{10}$ consider that the couplings of the heavy- and light-hole states at the minizone center due to interface and external-potential-inversion asymmetries play a much stronger role in the anisotropy than those due to the classical bulk-inversion asymmetry. Since the classical envelope function theory (EFT) does not take into account the details of interface bonding, people have found ways to include the interface effects into the envelope function framework: either through generalized boundary conditions as deduced by Ivchenko and Kaminski, ${ }^{2}$ or through a perturbed scheme called " $\mathrm{H}_{\mathrm{BF}}$ model" as introduced by Krebs and Voisin. ${ }^{3}$ They have the same form in describing the coupling, and the mixing coefficient, represented as $P$, can be considered as the magnitude of the potential localized at the interface (named as interface potential). Although tight-binding calculations can naturally take into account the full symmetry properties, it employs sophisticated computational methods that require extended running time, and the relative contributions to the anisotropy of different asymmetries are not easily discernible. EFT is more popular for its simple and reliable calculations. With the interface potential model, it is very easy and straightforward to discuss the interface effects on the optical properties of QW within EFT. Since the quality of the interfaces is important for optoelectronic devices, control the quality of heterointerfaces and study of atomic-scale microstructural features at interfaces are of interest. The in-plane optical anisotropy provides a new method of getting the information of buried interfaces through the value of $P$.

However, the origin of the interface potential is still under discussion. There is great divergence on the value of $P$ among the models. ${ }^{2,3,11-14}$ No systematic experiment has been done to testify them. One of the blockages is how to describe the coupling at $k=0$ due to the bulk term of the electric field, which can strongly influence the anisotropy especially for CA-QW (the well and barrier materials share a common anion, such as $\mathrm{GaAs} / \mathrm{Al}_{x} \mathrm{Ga}_{1-x} \mathrm{As}$ ). The point in dispute is the mixing coefficient. Zhu and Chang ${ }^{15}$ and Kwok et al. ${ }^{1}$ think that the mixing originates from converse piezoelectric effects, while Krebs et al. ${ }^{10,17}$ and Khurgin and Voision ${ }^{16}$ hold that it originates from the potential $e F z$ being incompatible with the cyclic boundary conditions used in the $\mathbf{k} \cdot \mathbf{p}$ theory. There is a five-times difference between the two models for $\mathrm{GaAs} / \mathrm{Al}_{x} \mathrm{Ga}_{1-x} \mathrm{As}$ interface. Very recently, Foreman ${ }^{18}$ has derived the coefficient by extending the formalism of Luttinger to higher order.

Until now, most of the works used polarized photoluminescence spectrum to study the anisotropy. Due to low sensitivity and relatively large error, it is very difficult to find out whether the allowed transitions of (001) CA-QW have in-plane anisotropy or not. However, CA-QW's are not so complex as NCA-QW's (the well and barrier materials share no common atom, such as $\operatorname{In}_{x} \mathrm{Ga}_{1-x} \mathrm{As} / \mathrm{InP} \mathrm{QW}$ ), since the left and the right interfaces are equivalent in CA-QW's and we need not deal with the additional electric field caused by the noncommutative band offset of NCA-QW's. Recently, the in-plane optical anisotropy (on $10^{-4}$ order) of the al- 


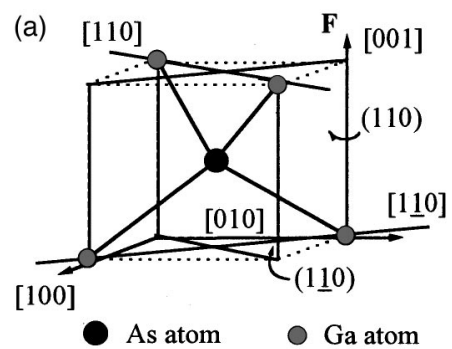

(b)

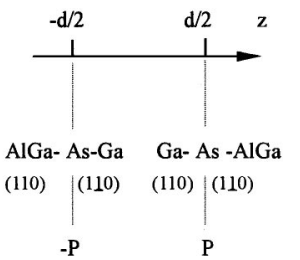

FIG. 1. (a) The coordinate system defined in calculations; (b) the definition of $P$ for the left and right interface in symmetric $\mathrm{GaAs} / \mathrm{Al}_{0.3} \mathrm{Ga}_{0.7} \mathrm{As}$, (110), etc., are the plane that the chemical bonds $\mathrm{As}-\mathrm{Al} /(\mathrm{Ga})$ lie in.

lowed and the forbidden transitions in $\mathrm{GaAs} / \mathrm{Al}_{x} \mathrm{Ga}_{1-x} \mathrm{As}$ and $\mathrm{In}_{x} \mathrm{Ga}_{1-x} \mathrm{As} / \mathrm{GaAs} \mathrm{QW}$ 's have been observed by reflectance-difference spectroscopy (RDS). ${ }^{7,23}$ RDS is designed to measure the small difference between the normalincidence complex reflectance for two orthogonal directions in the surface plane without rotating the polarizer or the sample. ${ }^{19}$ Its resolution can be as high as $10^{-5}$. One advantage of RDS is its easiness in analyzing the line shape. ${ }^{20}$ Generally, the values of the hole-mixing coefficients can be obtained by fitting the RDS curves. Therefore, with the help of RDS, GaAs/ $\mathrm{Al}_{x} \mathrm{Ga}_{1-x} \mathrm{As} \mathrm{QW}$ is the simplest system to study the hole-mixing effects.

This paper is devoted to analyzing relations among the line shape of RDS, the values of the mixing coefficients due to interface potential, and the electric field in (001) GaAs/ $/ \mathrm{Al}_{x} \mathrm{Ga}_{1-x} \mathrm{As}$ superlattices. We try to find out the most useful information from the measured RDS without doing tedious calculations for fitting the experimental curves. It will be a guide on how to measure the values of hole-mixing coefficients in (001) CA-QW's for future RDS experiments.

\section{CALCULATION DETAILS}

The envelope function theory is used to calculate the inplane anisotropic-transition strength in (001) $\mathrm{GaAs} / \mathrm{Al}_{x} \mathrm{Ga}_{1-x} \mathrm{As}$ superlattices for light propagating along [001] direction. The envelope functions and the energies of the electron and the hole are calculated by the method used in Ref. 15 except that we do not take into account the bulk inversion asymmetries, such as the linear $\mathbf{k}$ term, cubic $\mathbf{k}^{3}$ term, $a \mathbf{k}$ term, and the Rashba term. For hole states, in addition to $4 \times 4$ Luttinger Hamiltonian $H_{L},{ }^{21}$ a hole-mixing Hamiltonian $H^{\prime}$ is used to describe the couplings of heavyand light-hole states due to the interface potential and the bulk term of the electric field. In order to derive $H^{\prime}$, the coordination system is shown in Fig. 1(a), the definition of $P$ is show in Fig. 1(b). Regardless of the divergence in the theoretical models, the hole-mixing Hamiltonian $H^{\prime}$ has the form $^{3,7,11,14,17,18}$

$$
\begin{aligned}
H^{\prime}= & {\left[D F+p \sum_{n}\left[\delta\left(z-d_{1} / 2+n L\right)-\delta\left(z+d_{1} / 2+n L\right)\right]\right] } \\
& \times\left\{J_{x} J_{y}\right\}
\end{aligned}
$$

with

$$
\left\{J_{x} J_{y}\right\}=\left(\begin{array}{cccc}
0 & i & 0 & 0 \\
-i & 0 & 0 & 0 \\
0 & 0 & 0 & i \\
0 & 0 & -i & 0
\end{array}\right),
$$

where $d_{1}$ is the well width and $L$ is the period of the superlattice. The bases are the same as those of Luttinger and Kohn $;{ }^{21}$ the sequence is $\left|\frac{3}{2}, \frac{3}{2}\right\rangle ;\left|\frac{3}{2},-\frac{1}{2}\right\rangle ;\left|\frac{3}{2} \frac{1}{2}\right\rangle ;\left|\frac{3}{2},-\frac{3}{2}\right\rangle$. In Eq. (1), $D F$ represents the mixing coefficient due to electric field. For convenience, $D$ symbolizes different mixing models, equal to $d d_{14}=16.2 \times 10^{-10} \mathrm{eV} / \mathrm{cm} / \mathrm{V}$ for Zhu and Chang ${ }^{15}$ ( $d$ is the valence-deformation energy, $d_{14}$ is piezoelectric constant), and to $a / 4 \sqrt{3}=81.6 \times 10^{-10} \mathrm{eV} \mathrm{cm} / \mathrm{V}$ for Krebs et al. ${ }^{17}$ ( $a$ is lattice constant). If not stated specifically, $D_{0}$ $=16.2 \times 10^{-10} \mathrm{eV} \mathrm{cm} / \mathrm{V}$ is adopted in our calculations. Other parameters used ${ }^{15}$ are band-gap mismatch $\Delta E_{g}=1.155 x$ $+0.37 x^{2}$ ( $x$ is the composition of $\mathrm{Al}$ in $\mathrm{Al}_{x} \mathrm{Ga}_{1-x} \mathrm{As}$ ), $\Delta E_{c} / \Delta E_{v}=60: 40$, the band gap of GaAs $E_{g}=1.52 \mathrm{eV}$, Lutting parameters: $\gamma_{1}=6.85, \gamma_{2}=2.1, \gamma_{3}=2.9$.

Because the interface potential and the bulk term of electric field have a remarkable distinction from the bulk inversion asymmetries in that the former can generate in-plane anisotropy at $k=0$, so far almost all theoretical investigations are restricted to calculating the anisotropy at $k=0$. But the hole-mixing Hamiltonian is capable of coupling the heavyand light-hole states at $k \neq 0$, the anisotropy spectra contributed by all points in $k$ space are calculated in our work besides the computation at $k=0$. The dielectric function is expressed as

$$
\begin{aligned}
\varepsilon= & 1+\frac{\pi e^{2}}{m_{0}^{2} \omega^{2} \varepsilon_{0} V} \sum_{n, m} \sum_{k}\left|\left\langle\Phi_{n}|\overrightarrow{\mathbf{e}} \cdot \hat{p}| \Psi_{m}\right\rangle\right|^{2} \\
& \times\left\{\frac{1}{E_{n m}-E-i \Gamma}+\frac{1}{E_{n m}+E+i \Gamma}\right\},
\end{aligned}
$$

where $E_{n m}=E_{n}-E_{m}$ and the transition strength spectra is expressed as

$$
M=\sum_{n, m} \sum_{k}\left|\left\langle\Phi_{n}|\overrightarrow{\mathbf{e}} \cdot \hat{p}| \Psi_{m}\right\rangle\right|^{2} \delta\left(\hbar \omega-E_{n m}\right) .
$$

The calculations are restricted to band-to-band transitions. A Lorentzian function with $\Gamma=3 \mathrm{meV}$ is used to simulate the $\delta$ function in Eq. (2). Since excitonic effects are prominent in QW's, we assume that the excitonic features are polarized in the same way as the corresponding band-to-band transitions. ${ }^{10}$ The effects of the hole-mixing Hamiltonian on the excitons will be discussed later.

\section{DISCUSSIONS}

\section{A. Analysis of the reflectance-difference spectra}

Generally, we should fit the experimental RDS curves using various values of $P$ and $D$. But it is a time-consuming work because the divergence of the values of $P$ and $D$ is 
great, the excitonic effects must be taken into account, and the magnitude of the built-in field should be determined accurately. Now we try to find out if we can pick up some useful information from RDS without doing the tedious fitting work.

A GaAs/ $/ \mathrm{Al}_{x} \mathrm{Ga}_{1-x} \mathrm{As}$ superlattice sample consists of a GaAs substrate, a superlattice layer, and a cap layer. The normal-incidence complex reflectance $r$ for this system is given by

$$
r=\frac{V_{34}+r_{23} \exp \left(i 2 \varphi_{3}\right)}{1+V_{34} r_{23} \exp \left(i 2 \varphi_{3}\right)}
$$

with

$$
V_{i j}=\frac{\widetilde{n}_{j}-\widetilde{n}_{i}}{\widetilde{n}_{j}+\widetilde{n}_{i}}, \quad \varphi_{3}=\frac{2 \pi \widetilde{n}_{3} d_{3}}{\lambda},
$$

where $r_{23}$ is the complex reflectance at the boundary between the cap layer and the superlattice layer; the indices $i$ and $j$ take the values 1, 2, 3, 4 for the substrate, superlattice, cap, and external medium (in our case is the air), respectively; $\widetilde{n}_{i}$ is the complex refractive index of the $i$ th layer, $d_{3}$ is the thickness of the cap layer, $\lambda$ is the wavelength of the light. Since the bulk GaAs is isotropic and the surface is oxidized, the only potential anisotropy comes from the superlattice layer. By executing the differential calculations, we find that $\Delta r / r$ is proportional to the dielectric difference of the superlattice layer $\Delta \varepsilon=\varepsilon_{110}-\varepsilon_{1 \overline{1} 0}$,

$$
\begin{aligned}
\frac{\Delta r}{r} & =2 \times \frac{r_{110}-r_{1 \overline{1} 0}}{r_{110}+r_{1 \overline{1} 0}} \\
& =(\alpha+i \beta) \Delta \varepsilon \\
& \left.=\left(\alpha \Delta \varepsilon_{r}-\beta \Delta \varepsilon_{i}\right)+i\left(\alpha \Delta \varepsilon_{i}\right)+\beta \Delta \varepsilon_{r}\right)
\end{aligned}
$$

$\alpha$ and $\beta$ are complicated functions of the four refractive indices and the wavelength of the light. Equation (4) indicates that both the real and the imaginary components of $\Delta r / r$ are linear combinations of $\Delta \varepsilon_{r}$ and $\Delta \varepsilon_{i}$. If we assume that the transition matrix elements change slowly with wave vector $k$, which is a crude approximation for superlattice, then we get $\Delta \varepsilon_{i} \propto \Sigma_{n, m} \Delta M_{n m}(0) J_{n m}$, where $J_{n m}$ is the joint density of states, $\Delta \varepsilon_{r}$ has similar expression as $\Delta \varepsilon_{i}$. We can expect that the features of RDS are controlled by the anisotropy at $k=0$. Since we do not care about the absolute anisotropies of individual transitions at present, the line shape of the RDS is the most useful information, it may be determined by the anisotropic-transition strength ratios at $k=0$.

There is a special case for $d_{2} \ll \lambda$ where Eq. (4) reduces to

$$
\frac{\Delta r}{r}=\frac{4 \pi d_{2} \exp \left(i 2 \varphi_{3}\right)}{\lambda\left(\varepsilon_{1}-1\right)}\left(\Delta \varepsilon_{i}-i \Delta \varepsilon_{r}\right)
$$

if the thickness of the cap layer can be neglected when compared to the wavelength of the light, the real part of the RDS will be directly proportional to $\Delta \varepsilon_{i}$, which in turn is proportional to anisotropic-transition strength. We can compute $\Delta M$ only to directly compare with the experimental RDS.

\section{B. Anisotropic-transition strength ratio at $k=0$}

As typical RDS of $\mathrm{GaAs} / \mathrm{Al}_{x} \mathrm{Ga}_{1-x} \mathrm{As}$ superlattice has prominent features in the spectral range between $1 H 1 E$ $(1 \mathrm{HH} \rightarrow 1 \mathrm{CB}$ transition) and $1 L 1 E$, we will concentrate our effort on these two transitions. Though the calculation at $k$ $=0$ consumes little effort, we want to find out if $\Delta M_{1 H 1 E}(0) / \Delta M_{1 L 1 E}(0)$ has some relation to the $P / D$ ratio. The analytical two-level approach has the advantage of evidence. In practice, five lowest hole states are included in our calculation.

If there is a heavy-hole state and a light-hole state, their envelope functions and energies satisfy

$$
\begin{aligned}
& {\left[-\frac{\hbar^{2}}{2 m_{0}}\left(\gamma_{1}-2 \gamma_{2}\right) \frac{\partial^{2}}{\partial z^{2}}+V_{h}(z)-e F z\right] F_{h}=E_{h} F_{h},} \\
& {\left[-\frac{\hbar^{2}}{2 m_{0}}\left(\gamma_{1}+2 \gamma_{2}\right) \frac{\partial^{2}}{\partial z^{2}}+V_{h}(z)-e F z\right] F_{i}=E_{i} F_{i} .}
\end{aligned}
$$

The hole-mixing Hamiltonian, treated as a perturbation, will mix the two hole states and yield the anisotropic-transition strength

$$
\Delta M= \pm \frac{2}{\sqrt{3}} \frac{w f_{H E} f_{L E}}{\sqrt{\left(E_{L}-E_{H}\right)^{2}+4 w^{2}}}
$$

(if $E_{H}<E_{L}$, the sign " + " is for heavy-hole state, "_," for light hole state; while if $E_{H}>E_{L}$, the signs are reversed) where $f_{H E}$ (or $f_{L E}$ ) represents the overlap integral of the electron and the heavy-hole (or light-hole) envelope functions, $w$ is the mixing strength of the heavy- and light-hole states

$$
\begin{aligned}
w= & w_{1}+w_{2} \\
= & D F \int F_{H} F_{L} d z+P \sum_{n}\left[F_{H}\left(\frac{d_{1}}{2}+n L\right) F_{L}\left(\frac{d_{1}}{2}+n L\right)\right. \\
& \left.-F_{H}\left(-\frac{d_{1}}{2}+n L\right) F_{L}\left(-\frac{d_{1}}{2}+n L\right)\right],
\end{aligned}
$$

where $w_{1}$ is due to the bulk term of the field, $w_{2}$ is due to interface potential. Since the envelope functions approximately retain their parity at weak field, the bulk term of the field, being an even function of $z$, couples the heavy- and light-hole states of the same parity; while the interface potential, being an odd function, couples the states of opposite parity. Since $1 \mathrm{HH} ; 1 \mathrm{LH}, 2 \mathrm{HH}$ levels are close to each other but far away from the others, as to $1 L 1 E$

$$
\begin{aligned}
\Delta M_{1 L 1 E}= & -\frac{2}{\sqrt{3}} \frac{w_{1} f_{1 H 1 E} f_{1 L 1 E}}{\sqrt{\left(E_{1 L}-E_{1 H}\right)^{2}+4 w_{1}^{2}}} \\
& +\frac{2}{\sqrt{3}} \frac{w_{2} f_{1 L 1 E} f_{2 H 1 E}}{\sqrt{\left(E_{2 H}-E_{1 L}\right)^{2}+4 w_{2}^{2}}} \\
\cong & -\frac{2}{\sqrt{3}} \frac{w_{1} f_{1 H 1 E} f_{1 L 1 E}}{E_{1 L}-E_{1 H}}+\frac{2}{\sqrt{3}} \frac{w_{2} f_{1 L 1 E} f_{2 H 1 E}}{E_{2 H}-E_{1 L}} .
\end{aligned}
$$


In the last equation, it is assumed that $w_{1} \ll E_{1 L}-E_{1 H}$ and $w_{2} \ll E_{2 H}-E_{1 L}$. Because $w_{1} \propto D$ and $w_{2} \propto P$, Eq. (5) shows explicitly that the field and the interface potential mix the heavy- and light-hole states independently, which will give us a special benefit to find out the relation between the ratio $\Delta M_{1 H 1 E}(0) / \Delta M_{1 L 1 E}(0)$ and the ratio $P / D$.

At a given electric field, based on the values of $D_{0}$ and $P_{0}$ given by a certain model, it is easy to compute the anisotropic-transition strengths of the two transitions (represented by $A_{i}$ and $B_{i}$ for $1 H 1 E$ and $1 L 1 E$, respectively) for two special cases: (1) $D=D_{0}, P=0$; (2) $D=0, P=P_{0}$. Then for arbitrary $D$ and $P$, the corresponding anisotropictransition strengths (represented by $A$ and $B$ ) are the linear combinations of the two cases: $A=x A_{1}+y A_{2}$ and $B=x B_{1}$ $+y B_{2}$, with $D=x D_{0}, P=y P_{0}(x \geqslant 0)$; and for the sake of convenience, we introduce $A_{1} / B_{1}=-c, A_{1} / A_{2}=a, B_{1} / B_{2}$ $=b$.

Because $A_{i}, B_{i}$ and $A, B$ are functions of the electric field, $a, b$, and $c$ are functions of the field. We find that the anisotropy ratio and the ratio $P / D$ has the relation

$$
\begin{array}{r}
\frac{B}{A}=-\frac{x b+y}{x a+y} \frac{a}{c b}=-\frac{x_{0}+b}{x_{0}+a} \frac{a}{c b} \\
\text { or } x_{0}=-\frac{[c(B / A)+1] a b}{b c(B / A)+a}
\end{array}
$$

with $x_{0}=y / x$. With Eq. (9), it is convenient to obtain the ratio $\Delta M_{1 L 1 E} / \Delta M_{1 H 1 E}$ at arbitrary values of $P, D$ and at arbitrary electric field. It is evident that the ratio $\Delta M_{1 L 1 E} / \Delta M_{1 H 1 E}$ is determined by the ratio $P / D$. We can get some useful information from Eq. (9) (1) only if $-a$ $<x_{0}<-b$, then $\Delta M_{1 H 1 E}$ and $\Delta M_{1 L 1 E}$ have the same sign; (2) when $x_{0}=-a, \Delta M_{1 H 1 E}=0$, therefore as $x_{0}$ approaches to $-a, \Delta M_{1 L 1 E} / \Delta M_{1 H 1 E}$ will be much greater than 1; and for $x_{0}$ approaches to $-b, \Delta M_{1 H 1 E} / \Delta M_{1 L 1 E}$ will be much greater than 1 .

\section{Anisotropy contributed by all $k \neq 0$}

In this subsection we will present some qualitative analyses of whether the anisotropy contributed by all $k \neq 0$ changes the features at $k=0$ or not.

GaAs $/ \mathrm{Al}_{x} \mathrm{Ga}_{1-x} \mathrm{As}$ superlattices have similar valencesubband structure, as shown in Fig. 2. At points away from the zone center, $1 \mathrm{HH}$ level has an increasing light-hole component. According to Eq. (6), the light-hole component will decrease the anisotropy intensity of $1 \mathrm{HH}$ state. The heavyhole component of $1 \mathrm{LH}$ level does the same thing to $1 \mathrm{LH}$ state. That is, the anisotropy intensity will decrease as $k$ increases. Since the dispersion curves of 1HH and 1LH levels are quite flat around the zone center and are separated from the other levels, the anisotropy approximately maintains the value at $k=0$. Therefore, the anisotropy peak value can be written as $\Delta M(0) J^{\max }$, where the joint density of states (JDOS) $J$ is a steplike function and in an infinite quantum well every transition has an equal JDOS. But there is a peak at the edge of JDOS of $1 \mathrm{LH}$ level, it is estimated that 1

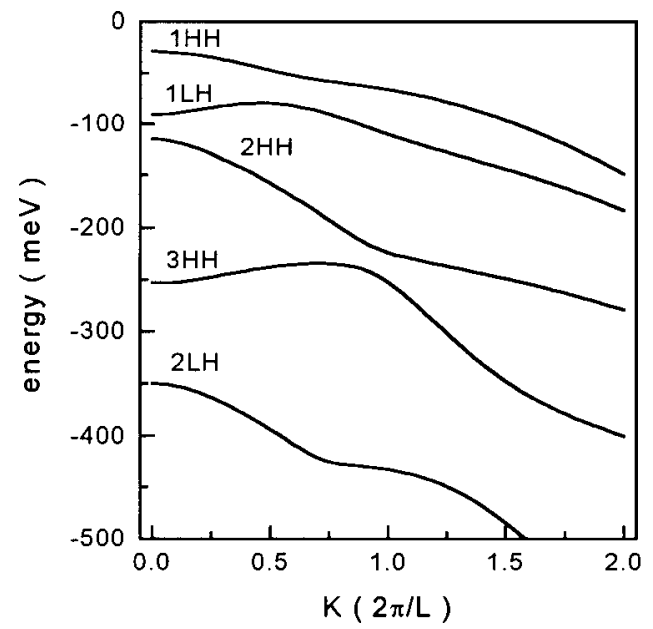

FIG. 2. Valence-band structure along the [100] direction in a $\operatorname{GaAs}(51 \AA) / \operatorname{AlAs}(51 \AA)$ superlattice.

$<J_{1 L 1 E}^{\max } / J_{1 H 1 E}^{\max }<1.5$. We can conclude that the anisotropy-peak ratio $\Delta M_{1 L 1 E} / \Delta M_{1 H 1 E}$ is governed by the same ratio at $k$ $=0$.

It should be noticed that the above argument is not suitable for all transitions, such as $2 \mathrm{H} 1 \mathrm{E}$ transition. Although $2 \mathrm{H} 1 \mathrm{E}$ has considerable anisotropy at $k=0$, the dispersion curve of $2 \mathrm{HH}$ level changes more quickly than $1 \mathrm{HH}$ and $1 \mathrm{LH}$ levels, and its strong coupling to $3 \mathrm{HH}$ state at $k \neq 0$ can change the feature of the anisotropy at $k=0$.

\section{COMPARISON WITH THE EXPERIMENTAL DATA}

A GaAs/AlAs superlattice is used for study. The sample is grown by MBE on a semi-insulating (001) GaAs substrate, after a $0.5 \mu \mathrm{m} \mathrm{GaAs}$ buffer layer is deposited, 60 periods of $\operatorname{GaAs}(51 \AA) / \operatorname{AlAs}(51 \AA)$ well/barrier are grown, then it is ended by a $200 \AA$ GaAs cap layer. Since it is intentionally undopped, the built-in electric field is weak. Its RDS (shown in Fig. 3) has typical line shape of $\mathrm{GaAs} / \mathrm{Al}_{x} \mathrm{Ga}_{1-x} \mathrm{As}$ superlattice: the imaginary part has one positive peak and one negative peak in the spectral range between $1 \mathrm{H} 1 \mathrm{E}$ and $1 \mathrm{~L} 1 \mathrm{E}$

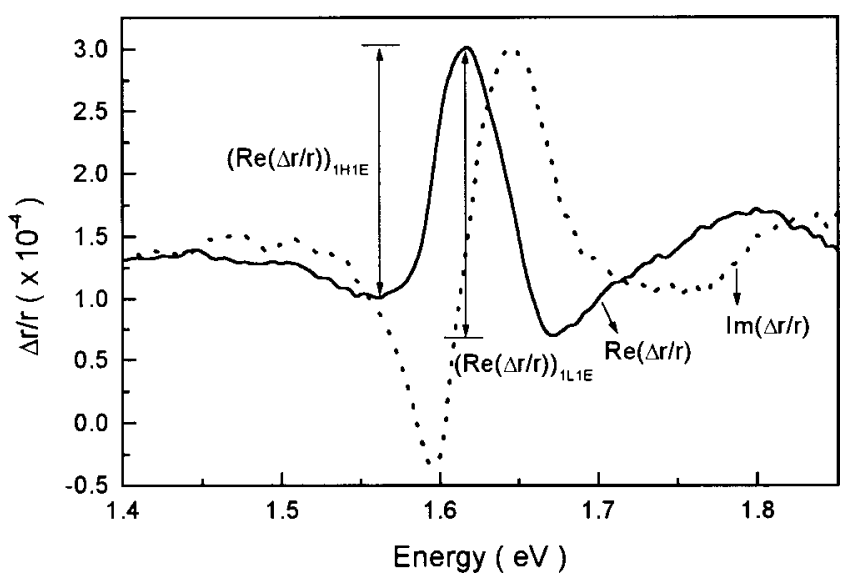

FIG. 3. Experimental reflectance-difference spectra of a $\operatorname{GaAs}(51 \quad \AA) / \operatorname{AlAs}(51 \quad \AA)$ superlattice measured at room temperature. 


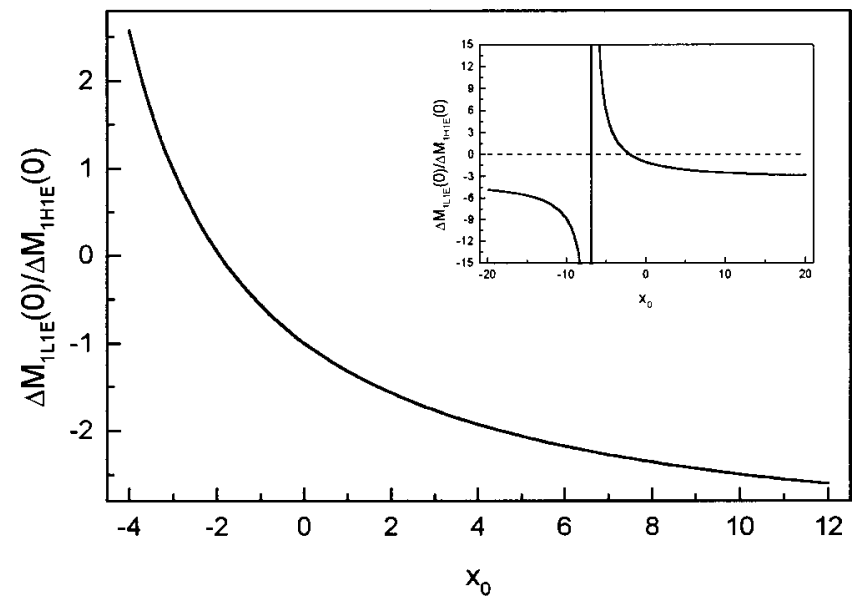

FIG. 4. Calculated changing tendency of the anisotropy intensity ratio $\Delta M_{1 L 1 E}(0) / \Delta M_{1 H 1 E}(0)$ versus the hole-mixing coefficients ratio $x_{0}=\langle P / D\rangle /\left\langle P_{0} / D_{0}\right\rangle$ under an field of $5000 \mathrm{~V} / \mathrm{cm}$ in $\operatorname{GaAs}(51$ $\AA) / \operatorname{AlAs}(51 \AA)$ superlattice with $D_{0}=16.2 \times 10^{-10} \mathrm{eV} \mathrm{cm} / \mathrm{V}, P_{0}$ $=14.6 \mathrm{meV} \AA$, shown in the inset is a complete picture.

transitions which implies that 1H1E and 1L1E have opposite anisotropy, and the line shape of the real part is almost like the derivative of the imaginary part. tios

From Fig. 3, we obtain the anisotropy-peak-intensity ra-

$$
\frac{\operatorname{Re}(\Delta r / r)_{1 L 1 E}}{\operatorname{Re}(\Delta r / r)_{1 H 1 E}}=-1.15, \quad \frac{\operatorname{Im}(\Delta r / r)_{1 L 1 E}}{\operatorname{Im}(\Delta r / r)_{1 H 1 E}}=-1.40 ;
$$

these two ratios are slightly different, but it shows clearly that the anisotropy of $1 \mathrm{~L} 1 \mathrm{E}$ is only slightly greater than that of $1 \mathrm{H} 1 \mathrm{E}$. For the line shape is not very sensitive to the anisotropy-peak ratio, we estimate that $\Delta M_{1 L 1 E}(0) / \Delta M_{1 H 1 E}(0)$ will be in the range $(-2,-1)$.

Now we know the range that $\Delta M_{1 L 1 E}(0) / \Delta M_{1 H 1 E}(0)$ is limited to, then we will work out the corresponding ratios $P / D$ by Eq. (9). As the field is weak, the anisotropy-intensity ratio no longer depends on the field strength. We take $F$ $=5 \times 10^{3} \mathrm{~V} / \mathrm{cm}$ as an example to perform the calculations. In the computations, we assume that $D_{0}=16.2$ $\times 10^{-10} \mathrm{eV} \mathrm{cm} / \mathrm{V}$, corresponding to the model of Zhu and Chang ${ }^{15}$ and Kwok et al. ${ }^{1}$ As to $P_{0}$, Krebs et al. ${ }^{17}$ have written the interface potential for GaAs/AlAs as $P_{0}$ $=a_{0} \Delta V / 2 \sqrt{3}=81.6 \mathrm{meV} \mathrm{nm}$; Chen et al. ${ }^{12}$ derived an interface potential from the interface strain as $P_{0}=d d_{14} V_{\mathrm{AHE}}$ $=1.46 \mathrm{meV} \mathrm{nm}\left(V_{\mathrm{AHE}}=0.09 \mathrm{eV}\right.$ for GaAs/AlAs, is the averaged hybrid-energy difference of the interface). We adopt $P_{0}=1.46 \mathrm{meV} \mathrm{nm}$ in this work. The changing tendency of the anisotropy-intensity ratio $\Delta M_{1 L 1 E}(0) / \Delta M_{1 H 1 E}(0)$ versus the hole-mixing-coefficient ratio $P / D$ is shown in Fig. 4. It is quickly obtained that $x_{0}$ should be in the range $(0,5)$.

To test the validity of our method, we have calculated the anisotropic-transition-strength spectra (shown in Fig. 5) contributed by all points in $k$ space $\left(k_{\|}<4 \pi / L\right)$ at the condition $D=D_{0}, \quad P=1.3 P_{0}$. It gives an anisotropic-transitionstrength ratio $\Delta M_{1 L 1 E} / \Delta M_{1 H 1 E}=1.2$ slightly smaller than the corresponding ratio at $k=0$ that is 1.4 . The detailed calculations demonstrate that the mixing at $k_{\|}=0$ indeed have

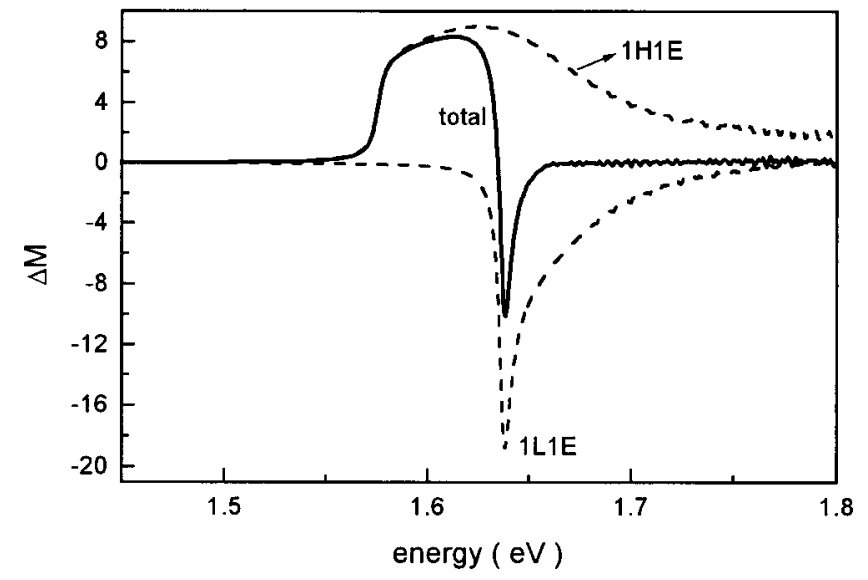

FIG. 5. Calculated anisotropic transition strength spectra of a $\operatorname{GaAs}(51 \AA) / \operatorname{AlAs}(51 \AA)$ superlattice with $D=16.2$ $\times 10^{-10} \mathrm{eV} \mathrm{cm} / \mathrm{V}, P_{0}=1.3 \times 14.6 \mathrm{meV} \AA, F=5000 \mathrm{~V} / \mathrm{cm}$.

contribution to the total anisotropy, the predominant portion comes from a smaller range of wave vector $k\left(k_{\|}<2 \pi / L\right)$; the averaged anisotropy near the zone center is almost the same as $\Delta M(0)$; when $k_{\|}$is far away from the center, the anisotropy can be quite different from that at $k=0$ due to complicated coupling. But for $1 \mathrm{H} 1 \mathrm{E}$ and $1 \mathrm{~L} 1 \mathrm{E}$ transitions the features of $\Delta M(0)$ are not affected by the large $k_{\|}$points. Though the individual anisotropy spectra of the two transitions partly overlap, the features of $\Delta M(0)$ still show evident embodiment in the spectra and the ratio of $\Delta M_{1 L 1 E} / \Delta M_{1 H 1 E}$ is similar to that at $k=0$.

Finally, we present the calculated RDS in Fig. 6. When $x_{0}=0$, only the imaginary part has similar line shape as the experiment [Fig. 6(a)], and it gives an anisotropy-peak ratio slightly smaller than the experiment. As $x_{0}$ increases, the real part of the calculated RDS becomes similar to the experimental curve. For $x_{0}=1.3$ [Fig. 6(b)], the calculated result agrees with the experimental line shape both for the real part and the imaginary part; if we take into account the excitonic effects, the anisotropy intensities will be improved by $5-10$ times $^{24}$ and the linewidth will agree with the experiment too. When $x_{0}$ is greater than 5 [Fig. 6(c)], both the two parts of the calculated RDS give an intensity ratio much larger than that from the experiment, and the calculated line shapes are quite different from the experiment.

Our method is proved to be efficient to determine the range that the ratio $P / D$ is limited to. The line shape of the RDS is determined by the ratio $P / D$. The exact values of $P$ and $D$ will not be achieved unless fitting the experiments. Even so, the determination of the range that $x_{0}$ limited to will greatly reduce the computational efforts, which is helpful for nondestructive probe of interfaces.

\section{GaAs/Al $\mathrm{Ga}_{x} \mathrm{Ga}_{1-x} \mathrm{As}$ SUPERLATTICES WITH HOMOGENEOUS IN-PLANE STRAIN}

In Bir-Pikus strained Hamiltonian, ${ }^{22}$ if only $\varepsilon_{x y} \neq 0$, then the strain Hamiltonian $H_{\varepsilon}$ has the same form as the holemixing Hamiltonian $H_{\varepsilon}=d \varepsilon_{x y}\left\{J_{x} J_{y}\right\}$. It implies that if there is a homogeneous in-plane strain in the symmetric 

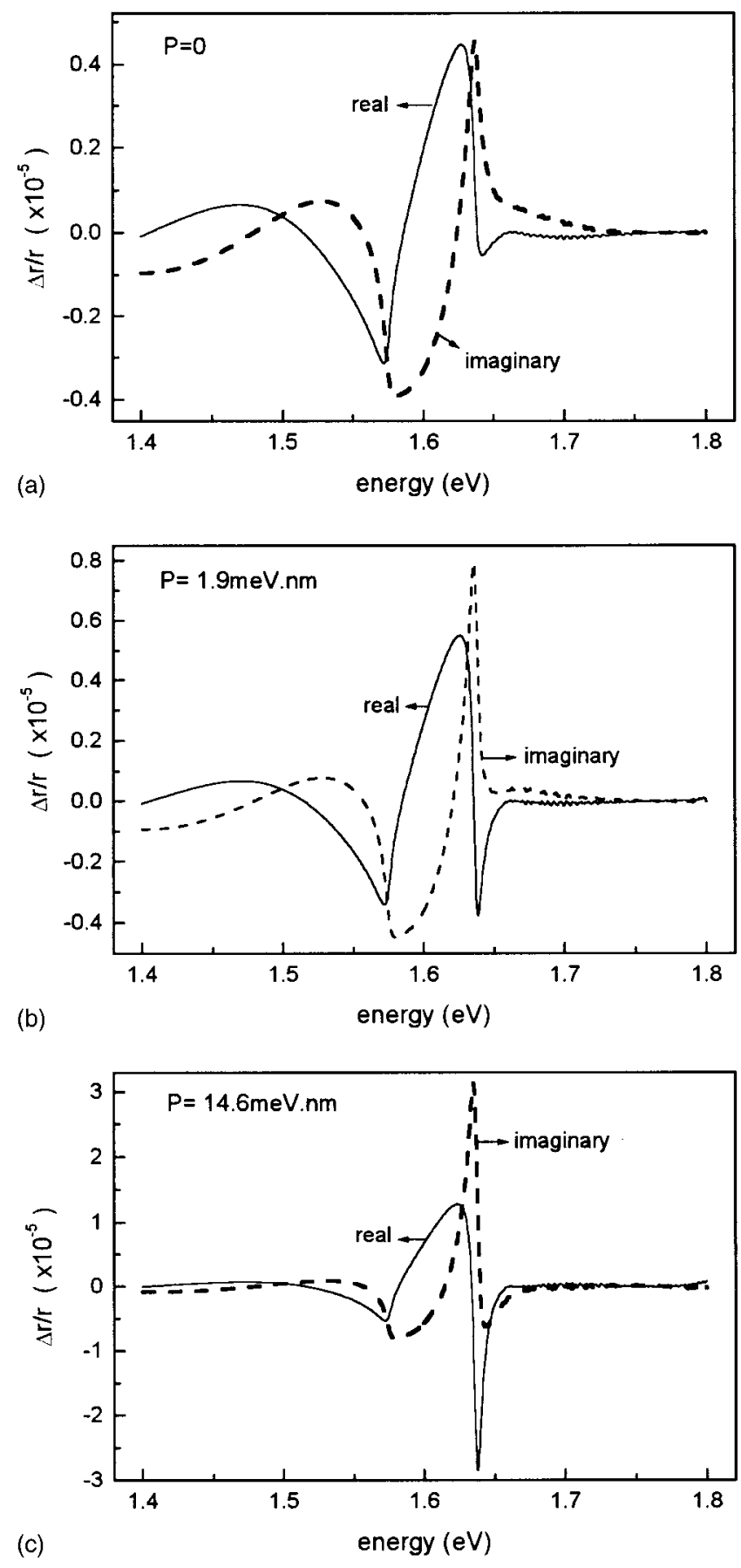

FIG. 6. Calculated reflectance-difference spectra (both the imaginary part and the real part) of a $\operatorname{GaAs}(51 \AA) / \operatorname{AlAs}(51 \AA)$ superlattice with different hole-mixing coefficient ratio $x_{0}$.

CA-QW's, the anisotropy cannot be zero even if there is no electric field. Similar to the bulk term of electric field, $H_{\varepsilon}$ couples the heavy- and light-hole states of the same parity, and a strain as small as $2.7 \times 10^{-6}$ can produce an anisotropy as large as that produced by a $10^{4} \mathrm{~V} / \mathrm{cm}$ field if we adopt $D=d d_{14}\left(d_{14}=-2.7 \times 10^{-10} \mathrm{~cm} / \mathrm{V}\right)$. If the hole-mixing strength due to the strain is much smaller than $\left|E_{\mathrm{H}}-E_{\mathrm{L}}\right|$, we find again that the strain, the electric field, and the interface potential mix the heavy- and light-hole states independently. It should be noticed that the anisotropy due to the strain can
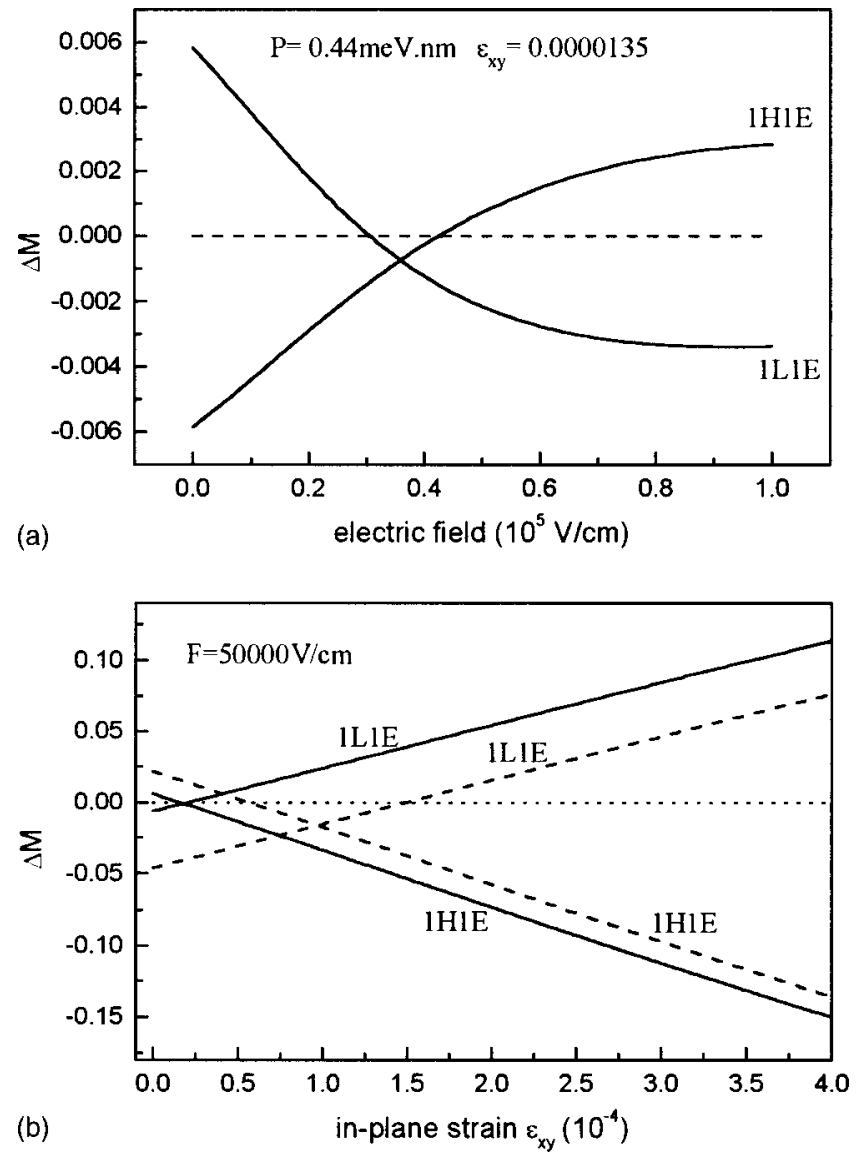

FIG. 7. (a) The field-dependent anisotropy at $k=0$ for $1 H 1 E$ and $1 L 1 E$ transitions with fixed in-plane strain; (b) the straindependent anisotropy at $k=0$ for $1 H 1 E$ and $1 L 1 E$ transitions with a fixed field in $\mathrm{GaAs} / \mathrm{Al}_{0.3} \mathrm{Ga}_{0.7} \mathrm{As}$ superlattice (solid lines are for $P=88 \mathrm{meV} \AA$, dash lines for $P=4.4 \mathrm{meV} \AA, \quad D=16.2$ $\times 10^{-10} \mathrm{eV} \mathrm{cm} / \mathrm{V}$.

be opposite to that due to the field, which may help us to determine the values of $D$ and $P$. Applying the same procedure as is used in Sec. III B, we additionally compute the anisotropic-transition strengths of $1 \mathrm{H} 1 \mathrm{E}$ (represented by $A_{3}$ ) and $1 \mathrm{~L} 1 \mathrm{E}$ (represented by $B_{3}$ ) at a fixed strain $\varepsilon_{x y}^{0}$ and a given electric field with $D=P=0$. For arbitrary strain $\varepsilon_{x y}$ $=z \varepsilon_{x y}^{0}$, we have $A=x A_{1}+y A_{2}+z A_{3}$ and $B=x B_{1}+y B_{2}$ $+z B_{3}$. If we alter the magnitude of the strain at a fixed field or alter the magnitude of the field at a fixed strain, we find that the anisotropic-transition strength can be tuned to zero (shown in Fig. 7). Instead of calculating the approximate value of $A / B$ at a fixed strain and the field, we can find out the magnitudes of the strain and the field satisfying $A=0$ or $B=0$ in the experiments and obtain a system of two linear equations ( $x$ and $y$ are the unknown parameters)

$$
\begin{aligned}
& x A_{1}\left(F_{1}\right)+y A_{2}\left(F_{1}\right)+z_{1} A_{3}\left(F_{1}\right)=0, \\
& x B_{1}\left(F_{1}\right)+y B_{2}\left(F_{1}\right)+z_{2} B_{3}\left(F_{1}\right)=0
\end{aligned}
$$

or 


$$
\begin{aligned}
& x A_{1}\left(F_{1}\right)+y A_{2}\left(F_{1}\right)+z_{1} A_{3}\left(F_{1}\right)=0, \\
& x A_{1}\left(F_{2}\right)+y A_{2}\left(F_{2}\right)+z_{2} A_{3}\left(F_{2}\right)=0 .
\end{aligned}
$$

The solution of either of these two systems will yield reliable values of $P$ and $D$. Liarokapis et al. ${ }^{24}$ have developed a new stress-inducing device to determine the electron and phonon deformation potential of thin layers and layered structures. The device can produce the in-plane strain that we want. They have found that RDS is very sensitive to small amounts of strain.

\section{CONCLUSIONS}

The in-plane anisotropy transition strengths in GaAs/ $/ \mathrm{Al}_{x} \mathrm{Ga}_{1-x} \mathrm{As}$ superlattice have been calculated within the envelope function theory, which includes the mixing of

*Corresponding author. FAX: +86-10-82305052. Email address: xlye@red.semi.ac.cn

${ }^{1}$ S. H. Kwok, H. T. Grahn, K. Ploog, and R. Merlin, Phys. Rev. Lett. 69, 973 (1992).

${ }^{2}$ E. L. Ivchenko and A. Yu. Kaminski, Phys. Rev. B 54, 5852 (1996).

${ }^{3}$ O. Krebs and P. Voisin, Phys. Rev. Lett. 77, 1829 (1996).

${ }^{4}$ O. Krebs, W. Seidel, J. P. Andre, D. Bertho, C. Jouanen, and P. Voisin, Semicond. Sci. Technol. 12, 938 (1997).

${ }^{5}$ R. Magri and S. Ossicini, Phys. Rev. B 58, R1742 (1998).

${ }^{6}$ L. C. Lew Yan Voon, Appl. Phys. Lett. 70, 2446 (1997).

${ }^{7}$ Y. H. Chen, Z. Yang, Z. G. Wang, Xu Bo, and J. B. Liang, Phys. Rev. B 60, 1783 (1999).

${ }^{8}$ A. V. Platonov, V. P. Kochereshko, and E. L. Ivchenko, Phys. Rev. Lett. 83, 3546 (1999).

${ }^{9}$ O. Krebs and P. Voisin, Phys. Rev. B 61, 7265 (2000).

${ }^{10}$ O. Krebs, R. Rondi, J. L. Centner, L. Goldstein, and P. Voisin, Phys. Rev. Lett. 80, 5770 (1998).

${ }^{11}$ B. A. Foreman, Phys. Rev. Lett. 81, 425 (1998).

${ }^{12}$ Chen Yong-hai, Wang Zhan-guo, and Yang Zhi-yu, Chin. Phys. Lett. 16, 56 (1999).

${ }^{13}$ E. L. Ivchenko, A. A. Toropov, and P. Voisin, Phys. Solid State 40, 1748 (1998). heavy- and light-hole states due to interface potential and electric field. Detailed analyses demonstrate that the range $P / D$ is limited to is determined by the line shape of RDS in the spectral range between $1 \mathrm{H} 1 \mathrm{E}$ and $1 \mathrm{~L} 1 \mathrm{E}$ transitions only. The calculations show that the reliable values of $P$ and $D$ will be achieved experimentally with the presence of a tunable in-plane strain $\varepsilon_{x y}$ or an electric field along [001] the direction.

\section{ACKNOWLEDGMENT}

This work was supported by the National Nature Science Foundation of China under Grant No. 69906003 and the special funds for Major State Basic Research Project No. G20000683 of China.

${ }^{14}$ E. E. Takhtaminov and V. A. Volkov, Phys. Low-Dimens. Semicond. Struct. 3/4, 203 (1999).

${ }^{15}$ B. F. Zhu and Y. C. Chang, Phys. Rev. B 50, 11932 (1994).

${ }^{16}$ J. B. Khurgin and P. Voision, Phys. Rev. Lett. 81, 3777 (1998); Semicond. Sci. Technol. 12, 1378 (1997).

${ }^{17}$ O. Krebs, P. Voisin, R. Rondi, J. L. Gentner, L. Goldstein, and J. C. Harmand, Physica E (Amsterdam) 2, 59 (1998).

${ }^{18}$ B. A. Foreman, Phys. Rev. Lett. 84, 2505 (2000).

${ }^{19}$ D. E. Aspnes, J. Vac. Sci. Technol. B 3, 1498 (1985); D. E. Aspnes, J. P. Harbison, and A. A. Studha, Phys. Rev. Lett. 59, 1687 (1987).

${ }^{20}$ Y. H. Chen, Z. Yang, Z. G. Wang, B. Ku, J. B. Liang, and J. J. Qian, Phys. Rev. B 56, 6770 (1997).

${ }^{21}$ J. M. Luttinger and W. Kohn, Phys. Rev. 97, 869 (1955).

${ }^{22}$ E. L. Evchenkov and G. E. Pikus, Superlattice and Other Heterostructures, translated by G. P. Skrebstrov (Springer-Verlag, Berlin, 1995).

${ }^{23}$ B. Koopman, P. V. Santos, and M. Cardona, Phys. Status Solidi A 170, 307 (1998).

${ }^{24}$ E. Liarokapis, D. Papadimitrious, J. Rumberg, and W. Richter, Phys. Status Solidi B 211, 309 (1999). 